\title{
Photocatalytic Activity of $\left(\mathrm{Ga}_{1-x} \mathrm{Zn}_{x}\right)\left(\mathrm{N}_{1-x} \mathrm{O}_{x}\right)$ for
}

\section{Visible-Light-Driven $\mathrm{H}_{2}$ and $\mathrm{O}_{2}$ Evolution in the Presence of}

\section{Sacrificial Reagents}

Kazuhiko Maeda, ${ }^{\dagger}$ Hiroshi Hashiguchi, ${ }^{\dagger}$ Hideaki Masuda, ${ }^{\dagger}$ Ryu Abe, ${ }^{*}$ and Kazunari Domen ${ }^{*}{ }^{\dagger}$

${ }^{\dagger}$ Department of Chemical System Engineering, The University of Tokyo, 7-3-1 Hongo, Bunkyo-ku,

Tokyo 113-8656, Japan. ${ }^{\ddagger}$ Catalysis Research Center, Hokkaido University, Sapporo 001-0021, Japan.

${ }^{\S}$ Research fellow of the Japan Society of Promotion Science (JSPS).

* To whom corresponding author should be addressed.

Department of Chemical System Engineering, The University of Tokyo,

7-3-1 Hongo, Bunkyo-ku, Tokyo 113-8656, Japan,

TEL: +81-3-5841-1148, FAX: +81-3-5841-8838

E-mail: domen@chemsys.t.u-tokyo.ac.jp 


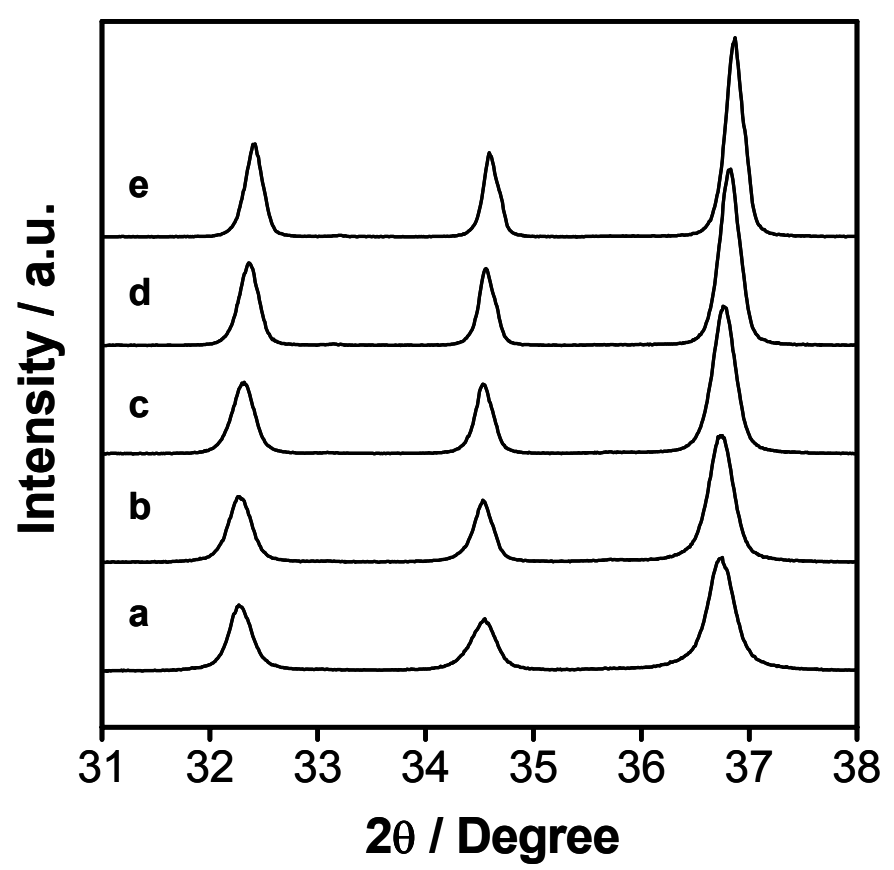

Figure S1. XRD patterns of samples (a)-(e)
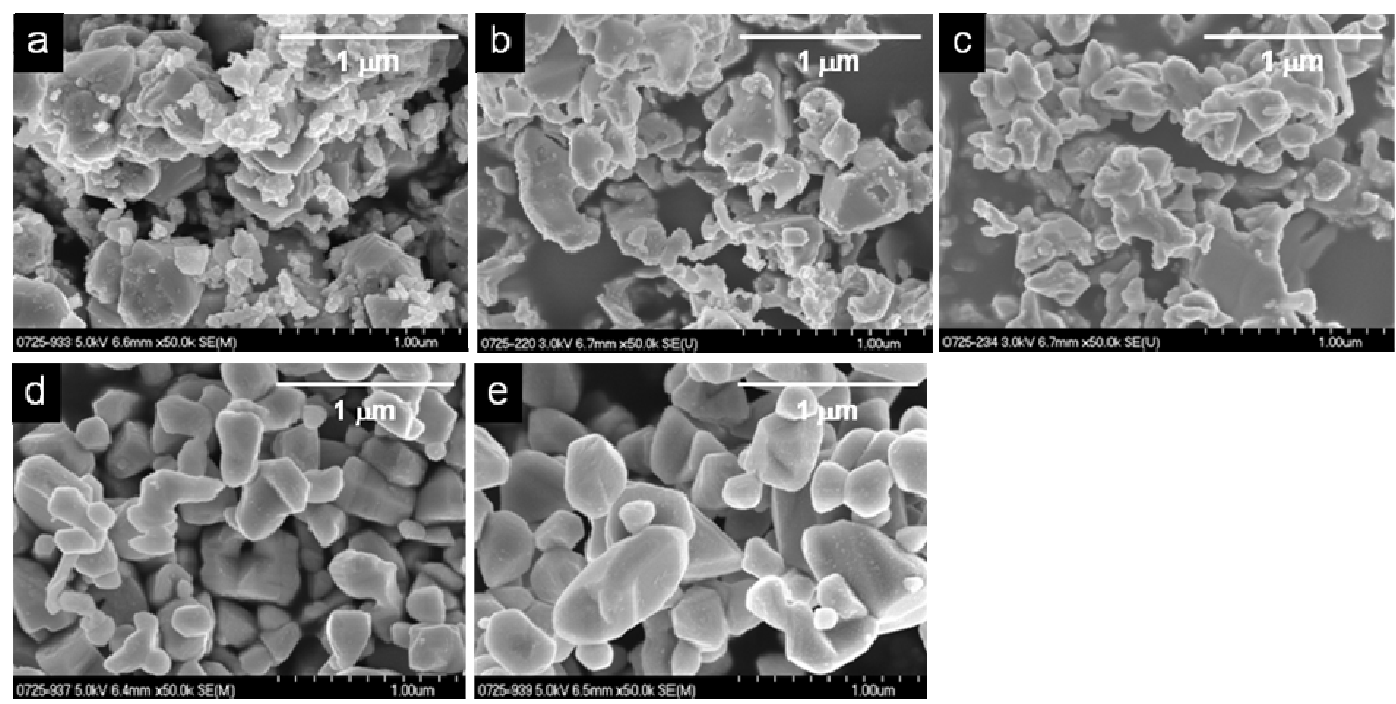

Figure S2. SEM images of samples (a)-(e) 


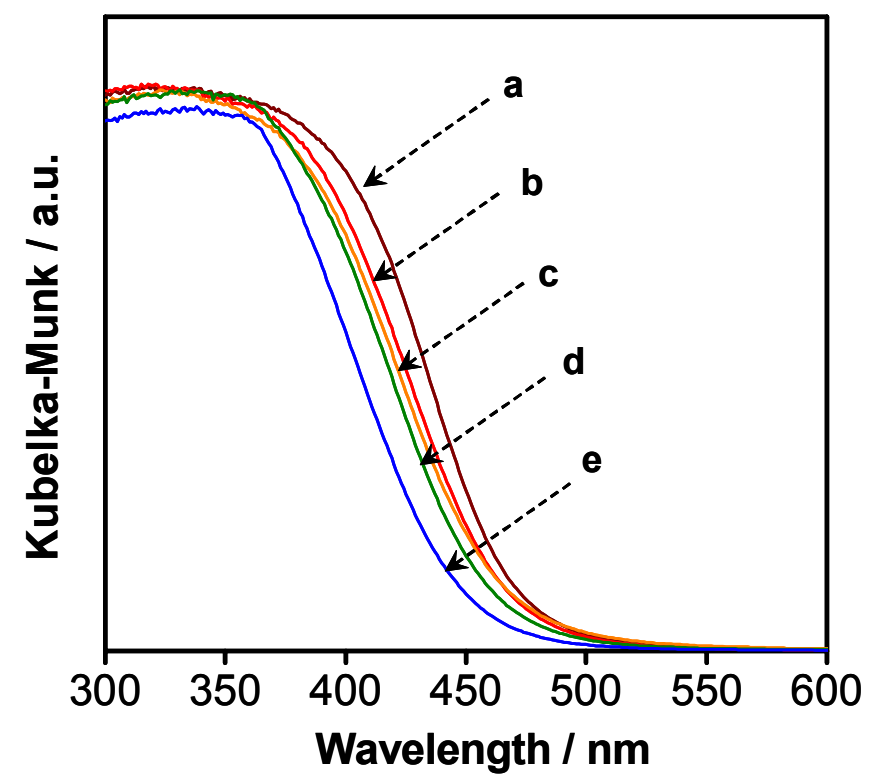

Figure S3. UV-vis. diffuse reflectance spectra for samples (a)-(e)

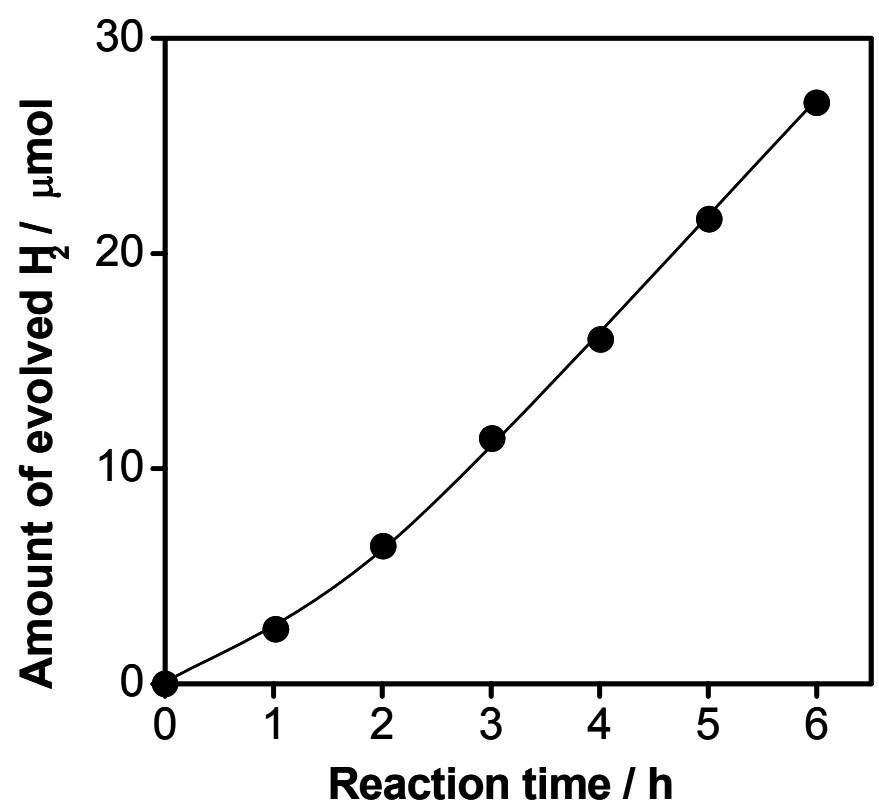

Figure S4. $\mathrm{H}_{2}$ evolution from an aqueous $\mathrm{NaI}$ solution $(0.01 \mathrm{M}, 100 \mathrm{~mL})$ by sample (f) loaded with $\mathrm{Rh}_{2-y} \mathrm{Cr}_{y} \mathrm{O}_{3}$ under visible light $(\lambda>420 \mathrm{~nm})$. Reaction conditions: catalyst, $0.1 \mathrm{~g}$; light source, xenon lamp (300 W) with cutoff filter; reaction vessel, Pyrex top-irradiation type. 